\title{
ASO Visual Abstract: Longitudinal Assessment of Quality of Life Following Molecular Testing for Indeterminate Thyroid Nodules
}

\begin{abstract}
Max A. Schumm, MD ${ }^{1}$, Dalena T. Nguyen, MPH ${ }^{1}$, Jiyoon Kim, $\mathrm{MSc}^{2}$, Chi-Hong Tseng, $\mathrm{PhD}^{\mathbf{3}}$, Amy Y. Chow, $\mathrm{MD}^{4}$, Na Shen, $\mathrm{MD}^{4}$, and Masha J. Livhits, $\mathrm{MD}^{1}$

${ }^{1}$ Section of Endocrine Surgery, Department of Surgery, University of California Los Angeles David Geffen School of Medicine, Los Angeles, CA; ${ }^{2}$ Department of Biostatistics, UCLA Fielding School of Public Health, Los Angeles, CA; ${ }^{3}$ Division of General Internal Medicine and Health Services Research, Department of Medicine, University of California Los Angeles David Geffen School of Medicine, Los Angeles, CA; ${ }^{4}$ Division of Endocrinology, Diabetes, and Metabolism, Department of Medicine, University of California, Los Angeles David Geffen School of Medicine, Los Angeles, CA
\end{abstract}

Patients with indeterminate thyroid nodules with benign molecular testing reported similar quality of life (QOL) through 18 months of surveillance, suggesting reassurance that the risk of malignancy is low (https://doi.org/10.1245/s10434-021-10375-6). Definitive surgery resulted in improvement of QOL in patients with suspicious test results.

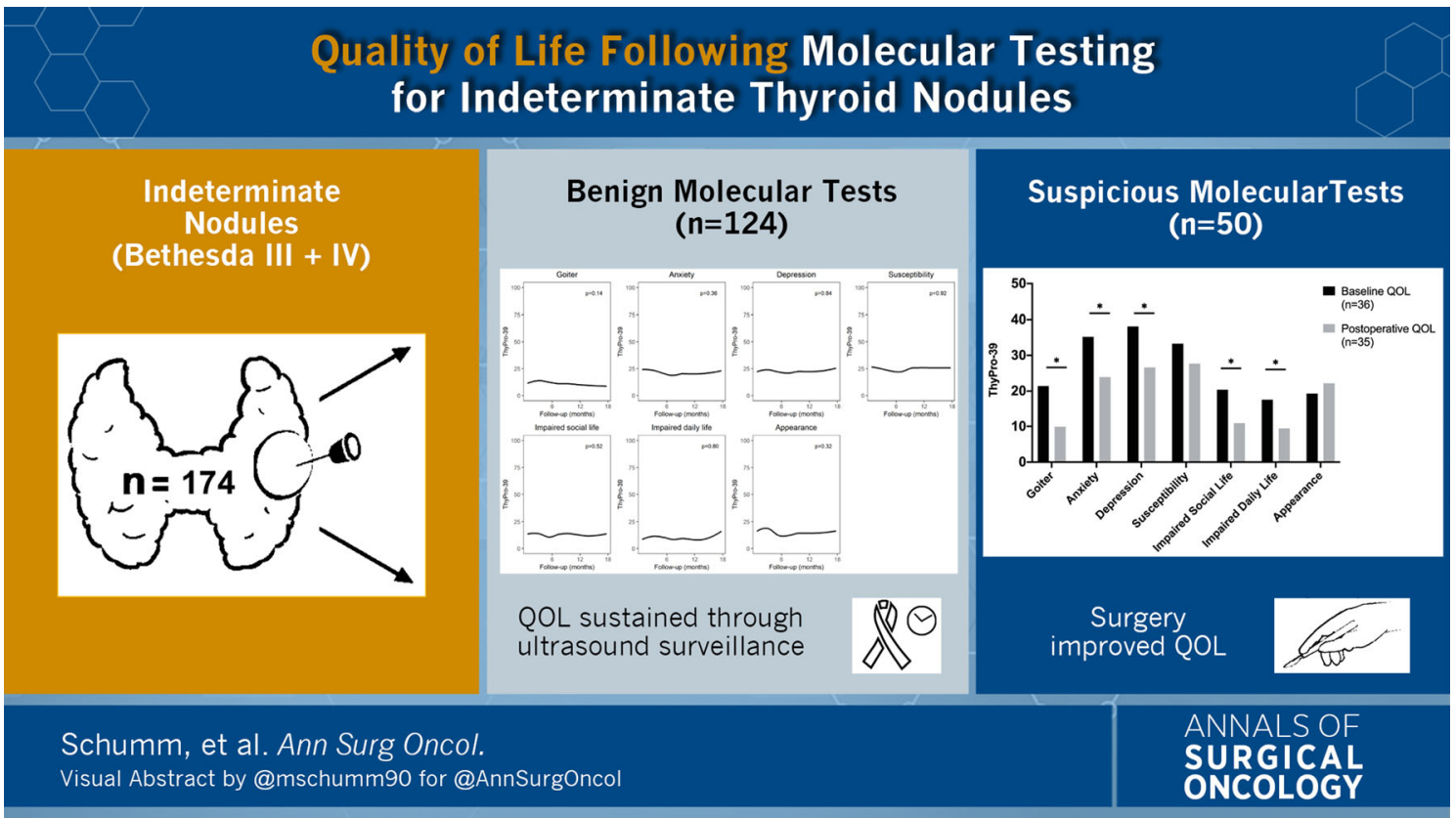

(C) Society of Surgical Oncology 2021

DISCLOSURE The authors have no related conflicts of interest to declare.

Published Online: 22 July 2021

M. A. Schumm, MD

e-mail: mschumm@mednet.ucla.edu

Publisher's Note Springer Nature remains neutral with regard to jurisdictional claims in published maps and institutional affiliations. 